\title{
Malignant Cerebral Edema Secondary to Gliadel Wafers in the Early Postsurgical Period
}

\author{
Ana B. Fernández¹, Antonio Garcia' ${ }^{1}$, Eglis Lazo² \\ ${ }^{1}$ Department of Anesthesiology, Intensive Care and Pain Treatment, Nuestra Sra de Candelaria University \\ Hospital, Santa Cruz de Tenerife, Spain \\ ${ }^{2}$ Department of Neurosurgery, Nuestra Sra de Candelaria University Hospital, \\ Santa Cruz de Tenerife, Spain \\ Email: anabfp@gmail.com
}

Received 20 February 2015; accepted 14 April 2015; published 15 April 2015

Copyright (C) 2015 by authors and Scientific Research Publishing Inc.

This work is licensed under the Creative Commons Attribution International License (CC BY). http://creativecommons.org/licenses/by/4.0/

(c) (i) Open Access

\begin{abstract}
High grade gliomas are the commonest intrinsic brain tumours and account for more average years of life lost than all the common cancers. It has become the commonest cause of cancer death in men under the age of 45 and women under the age of 35 . Although surgical resection can greatly reduce tumour bulk, complete excision is virtually impossible due to the infiltrative nature of these tumours. In an attempt to treat the infiltrating tumour cells, there has been much interest in using local therapies inserted at the time of surgery. The authors report a case of fatal cerebral edema unresponsive to aggressive medical and surgical assessment that finally evolved to premature death in the early postsurgical period, after the craniotomy and implantation of Gliadel wafers. They note that high doses of dexamethasone were insufficient to prevent cerebral edema and death. A search for corticosteroid use and dosing for patients treated with Gliadel wafers in the published literature revealed no recommendations on the doses of steroids to be administered. In our opinion this is a very important issue and maybe the key point for the treatment of this disease, and may need to be addressed with treatment guidelines in the near future in order to ensure better results on patient's survival. Prior to this case review there had been two similar report but a later presentation. So we think that this is the first case report of acute fulminant cerebral edema secondary to gliadel wafers in the early period.
\end{abstract}

\section{Keywords}

Malignant Glioma, Carmustine Wafer, Cerebral Edema, Cytotoxic Effect 


\section{Introduction}

Carmustine wafer (polifeprosan 20 with carmustine, Gliadel Wafers ${ }^{\circledR}$ ) is a sterile wafer that contains 192.3 mg of a biodegradable polyanhydride copolymer and $7.7 \mathrm{mg}$ of carmustine (1,3-bis (2-chlor-oethyl)-1-nitrosourea, or BCNU). BCNU wafers has been shown to improve survival in patients with newly diagnosed and recurrent malignant glioma (MG), and this survival benefit is maintained over the long term follow up in patients with newly diagnosed MG, but a variety of complications and adverse effects (AEs) have been associated including cerebral edema, intracranial hypertension, healing abnormalities, cerebral spinal fluid leaks infections seizures, thromboembolic complications hydrocephalus and cyst formation [1]. Following surgical resection, BCNU wafers are implanted into the resection cavity.

According to the literature currently available the overall incidence of cerebral edema in patients with newly diagnosed is $1.1 \%$. Rare cases of profound cerebral edema have been reported with standard doses of carmustine by weight [2] [3] but none has been reported in the early postsurgical period.

We describe a case of malignant cerebral edema unresponsive to aggressive medical and surgical assessment that finally evolved to premature death 3 days after the craniotomy and implantation of Gliadel wafers.

\section{Method}

59-year-old female was admitted to the hospital with a two week history of motor and sensory deficit on the left side, this increased in severity and a CT and MRI scan showed a mass lesion in the high right frontal-parietal area with minimal edema and greatest diameter of 2.7 - $3 \mathrm{~cm}$ (Figure 1). Dexamethasone started 4 mg every 8 h.

10 days after, a right frontal-parietal craniotomy was performed and the tumour was classified as a high-grade glioblastoma. Six Gliadel wafers were implanted on the brain surface. Immediately postoperation, patient presents neurological deterioration and worsens of left hemiparesis. A TC scan demonstrated a marked midline shift with edema throughout the right hemisphere more pronounced than on preoperative images. A drug regimen of $6 \mathrm{mg}$ dexamethasone and $20 \mathrm{~g}$ mannitol EV every $6 \mathrm{H}$ respectively, was initiated by improvement of the symptoms. $12 \mathrm{H}$ later, the patient began with a severe headache, a dense left hemiparesis, increased somnolence and she ceased to respond to painful stimuli.

A new CT scan demonstrated increased edema throughout the right hemisphere and further shift of the midline structure. Dexamethasone $10 \mathrm{mg}$ IV, mannitol 20 g every 4 h, and furosemide were given intravenously. Secondary to suddenly neurological decline and poorly response to aggressive medical treatment the reoperation was required.

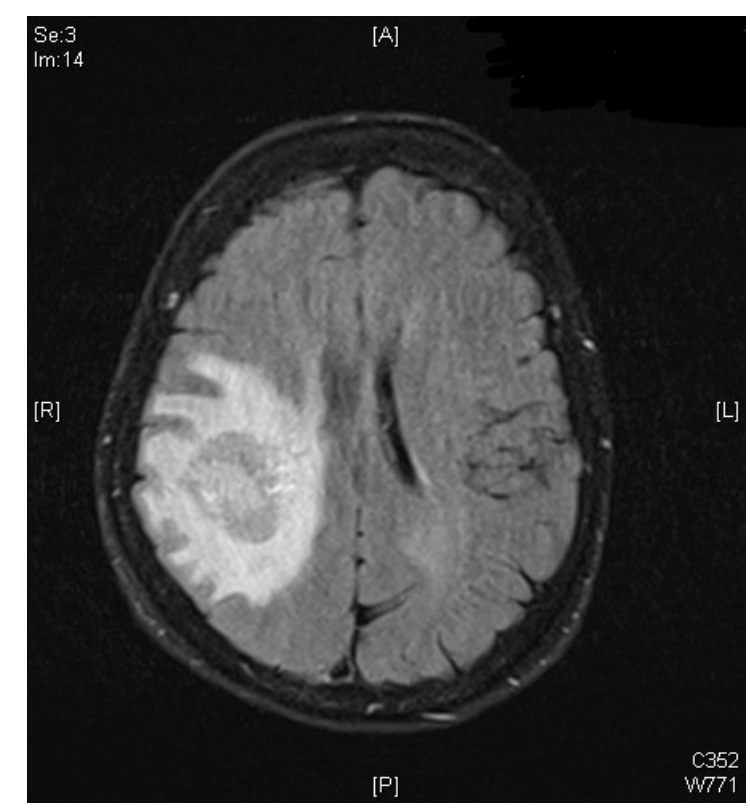

Figure 1. MRI scan: Mass lesion in the high right frontalparietal area. 


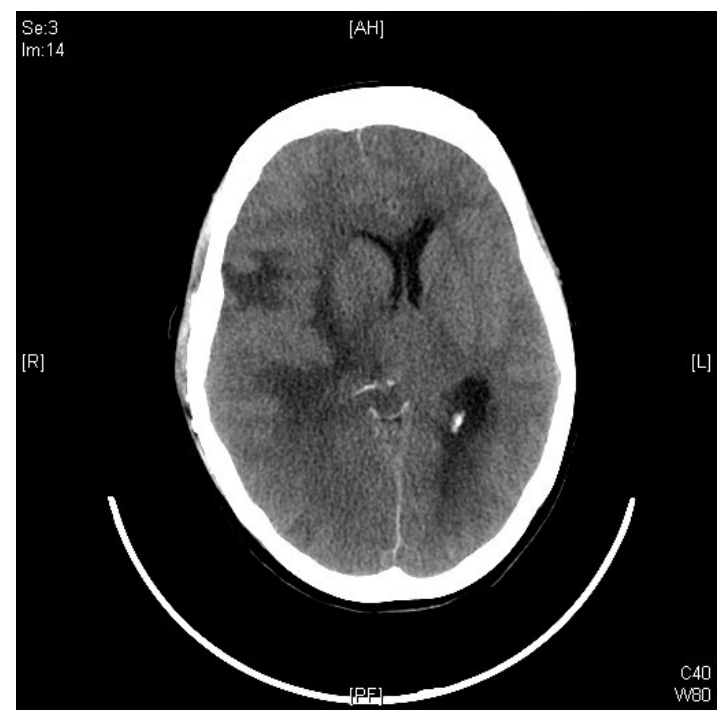

Figure 2. CT scan: Massive right uncal and subfalcine herniation.

\section{Results}

1 hour after surgery the patient was alert, oriented and responding appropriately with improvement in physical neurological exam. But in the next day she became progressively more obtunded, pupils became dilated, with Glasgow Coma Scale (GCS) score 2/15 and the patient died. A CT scan demonstrated massive right uncal and subfalcine herniation (Figure 2).

\section{Discussion}

While the use of Gliadel wafers for treatment of malignant gliomas has been shown in multiple studies to be effective with minimal adverse effects, we describe a early presentation of malignant cerebral edema in the inmediately postsurgical time which fits well with the potencial cytotoxic effects of carmustine. It has been shown that carmustine distributes widely, at least in the ipsilateral hemisphere, during the first several days postimplantation. Thus the inicial vasogenic edema induced by the surgery likely allowed for a situation of convective flow and greater distribution of carmustine with subsequent cytotoxicity. Fung et al. in studies on rats, concluded that carmustine distribution was increased in the first three days secondary to vasogenic edema and bulk flow [4] [5].

After revised the prior literature, we think that our patient is the first case of early malignant cerebral edema refractory to standard medical and surgical treatment secondary to Gliadel Wafers.

\section{References}

[1] Sabel, M. and Giese, A. (2008) Safety Profile of Carmustine Waters in Malignant Glioma: A Review of Controlled Trials and a Decade of Clinical Experience. Current Medical Research and Opinion, 24, 3239-3257. http://dx.doi.org/10.1185/03007990802508180

[2] Subach, B.R., Witham, T.F., Kondziolka, D., Lunsford, L.D., Bozik, M. and Schiff, D. (1999) Morbidity and Survival after 1,3-Bis(2-Chloroethyl)-1-Nitrosourea Wafer Implantation for Recurrent Glioblastoma: A Retrospective CaseMatched Cohort Series. Neurosurgery, 45, 17-22. http://dx.doi.org/10.1097/00006123-199907000-00004

[3] Weber, E. and Goebel, E. (2005) Cerebral Edema Associated with Gliadel Waters: Two Case Studies. Journal of NeuroOncology, 7, 84-89. http://dx.doi.org/10.1215/S1152851704000614

[4] Reulen, H.J., Graham, R., Spatz, M. and Klatzo, I. (1977) Role of Pressure Gradients and Bulk Flow in Dynamics of Vasogenic Brain Edema. Journal of Neurosurgery, 46, 24-35. http://dx.doi.org/10.3171/jns.1977.46.1.0024

[5] Fung, L.K., Shin, M., Tyler, B., Brem, H. and Saltzman, W.M. (1996) Chemotherapeutic Drugs Released from Polymers: Distribution of 1,3-Bis(2-Chloroethyl)-1-Nitrosourea in the Rat Brain. Pharmaceutical Research, 13, 671-682. http://dx.doi.org/10.1023/A:1016083113123 